\title{
Some Notes on Abundant Quasi-Ideals of Ample Semigroups
}

\author{
Chunhua $\mathrm{Li}^{1, \mathrm{a}}$ \\ ${ }^{1}$ School of Science, East China Jiaotong University, Nanchang 330013, China \\ achunhuali66@163.com
}

Keywords: natural partial orders; abundant quasi-ideals; (bi-)order ideals; ample semigroups.

Abstract. The aim of this paper is to study ample semigroups by using natural partial orders on abundant semigroups. After giving some properties and characterizations of abundant quasi-ideals on ample semigroups, we prove that there exists an idempotent-separating good congruence $\rho$ on an abundant quasi-ideal $M$ of an arbitrary ample semigroup $\mathrm{S}$, which can be extended to an idempotent-separating good congruence on S. In this paper, we develop an approach to the structure of ample semigroups inspired by the semigroup algebra theory of Mario petrich for inverse semigroups.

\section{Introduction}

The concepts of the natural partial orders on an abundant semigroup were introduced by Lawson [5] in 1987. After that, various classes of abundant semigroups are researched (see, [ 4, 7 ] )by considering the natural partial orders on abundant semigroup. In this paper, we shall study the IC-abundant semigroups by considering their natural partial orders and (bi-)order ideals. In particular, we will consider abundant quasi-ideals on ample semigroups. Some properties on IC-abundant semigroups and ample semigroups will be described in terms of their natural partial orders and their abundant quasi-ideals. We shall proceed as follows: section 2 provides some known results. In section 3, we give some characterizations of the abundant quasi-ideals on IC-abundant semigroups (e.g., IC-quasi-adequate semigroups). The last section we consider the abundant quasi-ideals and idempotent-separating good congruence extensions on ample semigroups.

\section{Preliminaries}

Here we provide some known results used repeatedly in the sequal. At first, we recall some basic facts about the relation $L^{*}$ and $R^{*}$.

LemmaII . $1^{[1]}$ Let $S$ be a semigroup and $a, b \in S$. Then the following statements are equivalent: (1) $a L^{*} b\left(a R^{*} b\right)$; (2) for all $x, y \in S^{1} a x=a y(x a=y a)$ if and only if $b x=b y(x b=y b)$.

CorollaryII .1 ${ }^{[1]}$ Let $S$ be a semigroup and $a, e=e^{2} \in S$.Then $a L^{*} e\left(a R^{*} e\right)$ if and only if $a=a e(a=e a)$ and for all $x, y \in S^{1} a x=a y(x a=y a)$ implies $b x=b y(x b=y b)$.

Evidently, $L^{*}$ is a right congruence while $R^{*}$ is a left congruence. For convenience, we denote by $a^{+}\left[a^{*}\right]$ a typical idempotent $R^{*}$ related $\left[L^{*}\right.$ related ] to $a$. $L_{a}^{*}\left(R_{a}^{*}\right)$ denotes the $L^{*}$ class $\left(R^{*}\right.$ class $)$ containing $a$. And $E(T)$ denotes the set of idempotents of $T ; \operatorname{Re} g(T)$ denotes the set of regular elements of $T$. We denote by $V(a)$ the set of all inverses of $a$. A semigroup $S$ is called abundant ${ }^{[3]}$ if and only if each $L^{*}$ class and each $R^{*}$ class contains at least one idempotent. An abundant semigroup $S$ is called quasi-adequate ${ }^{[2]}$ if its set of idempotents constitutes a subsemigroup (i.e., its set of idempotents is a band). Moreover, a quasi-adequate semigroup is called adequate ${ }^{[6]}$ if its bands of idempotents is a semilattice (i.e., the idempotents commute). An abundant semigroup $S$ is called ample $e^{[6]}$, if for all $e^{2}=e, a \in S, a e=(a e)^{+} a$ and $e a=a(e a)^{*}$. Follwing[1], an abundant semigroup $S$ is called idempotent-connected, for short, $I C$, provided for each $a \in S$ and for some $a^{+}, a^{*}$, there exists a bijection $\theta:\left\langle a^{+}\right\rangle \rightarrow\left\langle a^{*}\right\rangle$ such that $x a=a(x \theta)$ for all $\left.x \in<a^{+}\right\rangle$, where $\left\langle a^{+}\right\rangle$[resp. $\left.\left\langle a^{*}\right\rangle\right]$ is the subsemigroup of $S$ generated by the set $\left\{y \in E(S): y=y a^{+}=a^{+} y\right\}$ [resp. $\left\{y \in E(S): y=y a^{*}=a^{*} y\right\}$ ]. In fact, an ample semigroup is an IC-adequate semigroup and vice versa. Evidently, for an arbitrary adequate semigroup $S$, its each $L^{*}$ class and each $R^{*}$ class contains exactly one idempotent (i.e., $S$ is both $L^{*}$ - and $R^{*}$ - unipotent).

DefinitionII.$^{[5]}$ Let $S$ be an abundant semigroup. We define three relations on $S$, as follows:

(1) $a \leq_{r} b \Leftrightarrow R_{a}^{*} \leq R_{b}^{*}$, and there exists an idempotent $f \in R_{a}^{*}$ such that $a=f b$;

(2) $a \leq_{l} b \Leftrightarrow L_{a}^{* a} \leq L_{b}^{*}$, and there exists an idempotent $e \in L_{a}^{*}$ such that $a=b e$;

(3) $\leq=\leq_{r} \cap \leq_{l}$, i.e. $a \leq b \Leftrightarrow$ there exist idempotents $e, f$ such that $a=e b=b f$. 
Lemma II .2 ${ }^{[5]}$ Let $S$ be an abundant semigroup and $a, b \in S$. Then $a \leq_{r} b$ if and only if there exists $e \in R_{a}^{*} \cap E(S), f \in R_{b}^{*} \cap E(S)$ such that $a=e b$ and $e \leq f$.

Lemma II .3 ${ }^{[5]}$ Let $S$ be an abundant semigroup. Then $S$ is IC if and only if $s_{r}=\leq=s_{l}$.

Lemma II .4 ${ }^{[6]}$ Let $S$ be an ample semigroup, $a, b \in S$ and $a \leq b$. Then for all $c \in S$ $a c \leq b c, c a \leq c b, a^{+} \leq b^{+}$and $a^{*} \leq b^{*}$.

We recall from [1] that a semigroup homomorphism $\Phi: S \rightarrow T$ is a good homomorphism, if for all elements $a, b$ of $S, a L^{*}(S) b$ implies $a \Phi L^{*}(T) b \Phi$ and $a R^{*}(S) b$ implies $a \Phi R^{*}(T) b \Phi$. We say that a congruence $\rho$ on a semigroup $S$ is a good congruence if the natural homomorphism from $S$ onto $S / \rho$ is good. As defined in [9], a congruence $\rho$ on a semigroup $S$ is an idempotent-separating congruence, if for all $e, f \in E(S)$ the equality $e \rho=f \rho$ implies that $e=f$. From [1], we quote

Lemma II $.5^{[1]}$ Let $\rho$ be a congruence on an abundant semigroup $S$ and $a \in S$. Then $\rho$ is good if and only if $a \rho L^{*}(S / \rho) a^{*} \rho$ and $a \rho R^{*}(S / \rho) a^{+} \rho$.

Lemma II .6 ${ }^{[4]}$ Let $\rho$ be a good congruence on a quasi-adequate semigroup $S$. Then $\rho$ is idempotent-separating if and only if $\rho \subseteq H^{*}$.

Let $M$ be a subsemigroup of a semigroup $S$. We say that $M$ is a quasi-ideal of $S$ if $M S M \subseteq M$. A quasi-ideal $M$ is called abundant, if $M$ is an abundant semigroup. For an arbitrary semigroup $S$, a subset $F$ of the set of idempotents $E(S)$ is called order ideal of $E(S)$, if for all $f \in F, e \in E(S), e \leq f \Rightarrow e \in F$. In particular, if $\forall e, f \in F, \quad S(e, f) \neq \varnothing$, the we call $F$ a bi-order ideal of $E(S)$, where $S(e, f)=\{g \in E(S): e f=e g f, g e=g=f g\}$.

\section{Properties of abundant quasi-ideals on IC-quasi-adequate semigroups}

The aim of this section is to give some properties and characterizations of the natural partials on IC-quasi-adequate semigroups.

PropositionIII. 1 Let $S$ be an IC quasi-adequate semigroup and $Q$ a quasi-ideal of $S$. AQ denotes the set of abundant elements of $S$. Then the following statements are true:

(1) $E(Q)$ is a bi-order ideal of $E(S)$; $\quad$ (2) $A Q$ is an order ideal of $S$;

(3) $A Q$ is an abundant quasi-ideal of $S$; (4) $A Q$ is an IC-quasi-adequate semigroup.

Proof. (1) Let $e \in E(Q), f \in E(S)$ such that $f \leq e$. Then $f=e f=f e=e f e \in Q S Q \subseteq Q$. Hence $f \in E(Q)$. Suppose that $f, g \in E(Q)$. Since $S$ is a quasi-adequate semigroup, we have that $f g$ is an idempotent element of $S$. Let $x$ be an inverse element of $f g$. We can see that $g x f \in S(e, f) \cap E(Q)$.

Therefore, $E(Q)$ is a bi-order ideal of $E(S)$.

(2) We first prove that $A Q$ is an abundant subsemigroup of $S$. To see it, let $a, b \in A Q$. Then there are $a^{+}, b^{*} \in E(Q)$ such that $a R^{*}(Q) a^{+}$and $b L^{*}(Q) b^{*}$. Note that $a b L^{*}(S)(a b)^{*}$. Again since $L^{*}$ is a right congruence, we have $a b L^{*}(S)(a b)^{*} b^{*}$. Obviously, $(a b)^{*} b^{*} \in E(S),(a b)^{*} b^{*} \leq_{l} b^{*}$. Note that $S$ is IC. By Lemma II. 3, we have that $(a b)^{*} b^{*} \leq b^{*}$. Thus $(a b)^{*} b^{*} \in E(Q)$. Therefore, $a b L^{*}(Q)(a b)^{*} b^{*}$. Similarly, $a b R^{*}(Q) a^{+}(a b)^{+}$. This means that $A Q$ is an abundant subsemigroup of $S$.

Next, we prove that $A Q$ is an order ideal of $S$. To see it, let $x \in S, a \in A Q$ such that $x \leq a$. Then $x=e a=a f$, where $e \in E\left(R_{x}^{*}\right), f \in E\left(L_{x}^{*}\right)$. Hence $x=a^{+} e a$. Again since $Q$ is a quasi-ideal of $S$, we have $x \in Q$. On the other hand, since $R^{*}$ is a left congruence, we get that $x=a^{+} x R^{*}(S) a^{+} e$. Obviously, $a^{+} e \leq_{r} a^{+}$. Note that $S$ is IC. By Lemma II. 3, we have that $a^{+} e \leq a^{+}$. By (1), we have $a^{+} e \in E(Q)$ and $x R^{*}(Q) a^{+} e$. Similarly, $x L^{*}(Q) f a^{*}$, where $f a^{*} \in E(Q)$. Therefore, $x \in A Q$. That is, $A Q$ is an order ideal of $S$.

(3) Let $a, b \in A Q, x \in S$. Then $a x b \in Q$. On the other hand, $a x b L^{*}(S)(a x b)^{*}$. Since $L^{*}$ is a right congruence, we have that $a x b L^{*}(S)(a x b)^{*} b^{*}$. Obviously, $(a x b)^{*} b^{*} \leq_{l} b^{*}$. Note that $S$ is IC. By Lemma II. 3, we get $(a x b)^{*} b^{*} \leq b^{*}$. By (1), $(a x b)^{*} b^{*} \in E(Q)$. Therefore, $a x b L^{*}(Q)(a x b)^{*} b^{*}$. 
Similarly, $a x b R^{*}(Q) a^{+}(a x b)^{+}$and $a^{+}(a x b)^{+} \in E(Q)$. Therefore, $a x b \in A Q$. This means that $A Q$ is an abundant quasi-ideal of $S$.

(4) It follows from (3) and the definition of IC.

Theorem III.1 Let $S$ be an IC-quasi-adequate semigroup and $F$ be a bi-order ideal of $E(S)$. Put $M=\left\{a \in S: a^{*} \in F \cap L_{a}^{*}, a^{+} \in F \cap R_{a}^{*}\right\}$. Then $M$ is an abundant quasi-ideal of $S$ with $F=E(M)$, and $M=\bigcup\{u S v: u \in F, v \in F\}$.

Proof. Let $F$ be a bi-order ideal of $E(S)$. Then for all $a, b \in M, x \in S$, $a x b L^{*}(S)(a x b)^{*}$. Since $L^{*}$ is a right congruence, we have that $a x b L^{*}(S)(a x b)^{*} b^{*}$. Obviously, $(a x b)^{*} b^{*} \leq_{l} b^{*}$. Note that $S$ is IC. By Lemma II. 3, we have that $(a x b)^{*} b^{*} \leq b^{*}$, and so $(a x b)^{*} b^{*} \in F$. Similarly, we can prove that $a x b R^{*}(S) a^{+}(a x b)^{+}$, and $a^{+}, a^{+}(a x b)^{+} \in F$. Therefore, $a x b \in M$. That is, $M S M \subseteq M$. Obviously, $M$ is abundant. This means that $M$ is an abundant quasi- ideal of $S$.

Next, we prove that $F=E(M)$. It is clear that $F \subseteq E(M)$. We only need to prove $F \supseteq E(M)$. To see it, let $e \in E(M)$. Then there exist idempotents $e^{*}, e^{+} \in F$ such that $e^{+} R^{*}(M) e L^{*}(M) e^{*}$. But $S$ is an ample semigroup. We have $e=e^{+}=e^{*} \in F$. This gives that $F=E(M)$.

Now, we prove that $M=\bigcup\{u S v: u \in F, v \in F\}$. We only need to prove inverse inclusion. Let $a \in u S v$, where $u, v \in F$. Then there is $x \in S$ such that $a=u x v$. Note that $u x L^{*}(S)(u x)^{*}$. We have that $u x v L^{*}(S)(u x)^{*} v$ since $L^{*}$ is a right congruence. Obviously, $(u x)^{*} v \leq_{l} v$. Again since $S$ is IC, by Lemma II. 3, $(u x)^{*} v \leq v$, and so $(u x)^{*} v \in F$. Similarly, $u x v R^{*}(S) u(x v)^{+} \in F$. Therefore, $u x v \in M$. This completes the proof.

\section{Abundant quasi-ideals on an ample semigroup}

In this section, we will consider abundant quasi-ideals on ample semigroups.

Let $R$ be a relation on a semigroup $S, a, b \in S$ and $N$ denote the set of all non-negative integers. We define a relation as follows:

$$
(a, b) \in R^{\#} \Leftrightarrow a=b \text { or } a=z_{1} \rightarrow z_{2} \rightarrow \cdots \rightarrow z_{n-1} \rightarrow z_{n}=b \quad(n \in N)
$$

Where $z_{i} \rightarrow z_{i+1}$ denotes $z_{i}=u_{i} a_{i} v_{i}, z_{i+1}=u_{i} b_{i} v_{i}$, and $\left(a_{i}, b_{i}\right) \in \rho(1 \leq i \leq n)$. We call the above relation a Sequence of elementary $R$ - transitions connecting $a$ to $b^{[8]}$.

Theorem IV.1 Let $S$ be an ample semigroup. Then the following statements are true:

(1) for all $e, f \in E(S)$, eSf is a quasi-ideal of $S$;

(2) if $e S f$ is an abundant quasi-ideal of $S$ for all $e, f \in E(S)$, then $e S f$ is an ample semigroup, and $a^{*}=a^{*} e, a^{+}=f a^{+}$for all $a \in e S f$;

(3) if eSf is an abundant quasi-ideal of $S$ for all $e, f \in E(S)$, then a congruence $\rho$ on $e S f$ can be extened to a congruence on $S$.

Proof. (1) This is trivial. (2) Let eSf be an abundant quasi-ideal of $S$ for all $e, f \in E(S)$. Then, by Proposition III.1, it is clear that eSf is ample. Let $a \in e S f$. Then $a L^{*}(e S f) a^{*}$, and $a R^{*}(e S f) a^{+}$. Hence $a^{*}=e a^{*}=a^{*} e, a^{+}=a^{+} f=f a^{+}$.

(3) Let $e S f$ be an abundant quasi-ideal of $S$ for all $e, f \in E(S)$ and let $\rho$ be a congruence on $e S f$. Denote $\rho^{\#}$ a congruence on $S$ generated by $\rho$. We only need to prove $\rho=\rho^{\#} \cap e S f \times e S f$. Obviously, $\rho \subseteq \rho^{\#} \cap e S f \times e S f$. Conversely, let $(x, y) \in \rho^{\#} \cap e S f \times e S f$. Then either $x=y$ or for some $n$ in $N$ there is a sequence $x=x_{1} \rightarrow x_{2} \rightarrow \cdots \rightarrow x_{n}=y$ such that $x_{i}=u_{i} a_{i} v_{i}, x_{i+1}=u_{i} b_{i} v_{i}$, where $u_{i}, v_{i} \in S^{1},\left(a_{i}, b_{i}\right) \in \rho \quad(1 \leq i \leq n)$. Again since $x=e x f, y=e y f$, we have

$$
x=e x_{1} f \rightarrow e x_{2} f \rightarrow \cdots \rightarrow e x_{n} f=y
$$


By (2), ex $f=e u_{i} a_{i} v_{i} f=e u_{i} a_{i}^{+} a_{i} a_{i}^{*} v_{i} f=e u_{i} f a_{i}^{+} a_{i} a_{i}^{*} e v_{i} f=\left(e u_{i} f\right) a_{i}\left(e v_{i} f\right)$. Similarly, we have $e x_{i+1} f=e u_{i} b_{i} v_{i} f=\left(e u_{i} f\right) b_{i}\left(e v_{i} f\right)$. But $\left(a_{i}, b_{i}\right) \in \rho$ and $e u_{i} f, e v_{i} f \in e S f$. Thus $\left(e x_{i} f, e x_{i+1} f\right) \in \rho$, $(1 \leq i \leq n)$. Therefore, $(x, y) \in \rho$. The proof is completed.

Theorem IV.2 Let $M$ be an abundant quasi-ideal on an ample semigroup $S$ and $\rho$ an idempotent-separating good congruence on $M$. Then the following statements are true:

(1) $a \rho b \Rightarrow a^{*}=b^{*}, a^{+}=b^{+}(\forall a, b \in M)$;

(2) if $\rho=1_{M}$, then $\rho^{\#}$ is an idempotent-separating good congruence on $S$;

(3) if $\rho^{\#}$ is an idempotent-separating good congruence on $S$, then $\rho=\rho^{\#} \cap M \times M$.

Proof. (1) Note that $S$ is an ample semigroup. By Proposition III.1, $M$ is an ample subsemigroup of $S$. Let $a, b \in M$ such that $a \rho b$. Then, by Lemma II.6, $\rho \subseteq H^{*}(M) \subseteq L^{*}(M)$. Hence $a L^{*}(M) b$, and so $a^{*}=b^{*}$. Similarly, $a^{+}=b^{+}$. (2) It is clear. (3) Firstly, we note that $(s, t) \in \rho^{\#}$ for all $s, t \in S$ if and only if either $s=t$ or there exists a sequence $s=s_{1} \rightarrow s_{2} \rightarrow \cdots$ $\rightarrow s_{n}=t \quad(n \in N), \quad$ where $s_{i} \rightarrow s_{i+1} \quad$ means that $s_{i}=u_{i} a_{i} v_{i}, s_{i+1}=u_{i} b_{i} v_{i}$ and $\left(a_{i}, b_{i}\right) \in \rho$ $(1 \leq i \leq n)$. Suppose that $s_{i} \in M$, we prove that $s_{i+1} \in M$ and $s_{i}, s_{i+1}$ are $\rho$ related. Since $\rho$ and $\rho^{\#}$ are idempotent-separating good congruences on $M$ and $\left(a_{i}, b_{i}\right) \in \rho$, we have that $s_{i+1}^{*}=s_{i}^{*}, s_{i+1}^{+}=s_{i}^{+}$,

$a_{i}^{*}=b_{i}^{*}$ and $a_{i}^{+}=b_{i}^{+}$. Hence $s_{i+1}=s_{i}^{+} s_{i+1} s_{i}^{*}=s_{i}^{+} u_{i} a_{i}^{+} b_{i} a_{i}^{*} v_{i} s_{i}^{*}=\left(s_{i}^{+} u_{i} a_{i}^{+}\right) b_{i}\left(a_{i}^{*} v_{i} s_{i}^{*}\right)$. Since $M$ is an abundant quasi-ideal of $S$, we have that $w_{i}=\left(s_{i}^{+} u_{i} a_{i}^{+}\right) \in M S M \subseteq M, z_{i}=\left(a_{i}^{*} v_{i} s_{i}^{*}\right) \in M S M \subseteq M$. Thus $s_{i+1} \in M$. But $\left(a_{i}, b_{i}\right) \in \rho$, we have $\left(w_{i} a_{i} z_{i}, w_{i} b_{i} z_{i}\right) \in \rho$. That is $\left(s_{i}, s_{i+1}\right) \in \rho$. Therefore, if $s \in M$ and $(s, t) \in \rho^{\#}$, then $t \in M$ and $(s, t) \in \rho$. That is, $\rho^{\#} \cap M \times M \subseteq \rho$. The reverse containment is clear. Therefore, $\rho=\rho^{\#} \cap M \times M$.

\section{Conclusions}

In this paper, we investigate abundant quasi-ideals on IC-abundant semigroups and give some properties and characterizations of abundant quasi-ideals on ample semigroups by using the natural partial orders on abundant semigroup.

\section{Acknowledgements}

This work is supported by the National Science Foundation ( China ) ( No. 11261018), and the Science Foundation of the Education Department of Jiangxi Province (No. GJJ14381; KJLD12067).

\section{References}

[1] A. El-qallali and J. B. Fountain: Proc. Roy. Soc. Edinburgh vol.91(1981), p. 79.

[2] A. El-qallali and J. B. Fountain: Proc. Roy. Soc. Edingburgh. Sect. vol. 91(1981), p. 91.

[3] J. B. Fountain: Proc. London Math. Soc. vol.44(1982), p.103.

[4] Guo Xiaojiang: Jour. Pure Math vol. 16(1999), p. 57.

[5] M. V. Lawson: Proc. Edinburgh Math. Soc. vol. 30(1987), p.169.

[6] M. V. Lawson: Quart. J. Math. Oxford. vol.37(1986), p. 279.

[7] Chunhua Li, Limin Wang : Semigroup Forum vol.82(2011), p.516.

[8] M. Petrich. Completely regular semigroups ( New York: Jhon Wiley\&Sons Inc,1999 ).

[9] M. Petrich. Inverse semigroups ( New York: Jhon Wiley\&Sons Inc,1984 ). 